\title{
Rosai-Dorfman Disease Confined to the Breast
}

\author{
Thais Helena de Souza Silotti, ${ }^{\mathrm{a}, \mathrm{c}}$, Maria Riselda Vinhote da Silva ${ }^{\mathrm{a}}$, \\ Nilton Cesar O Bessa ${ }^{\mathrm{b}}$, Ione Rodrigues Bruna, \\ Livia Moura de Oliveira ${ }^{a}$
}

\begin{abstract}
The Rosai-Dorfman (also known as sinus histiocytosis with massive lymphadenopathy), was described in 1969 by Rosai and Dorfman, and is considered a rare disease of unknown aetiology characterized by benign histiocytic lymphadenopathy in the neck and extranodal sites. The involvement of the breast is rare and often simulates invasive breast carcinoma in its clinical presentation and radiological findings. There are currently about 20 cases reported in the literature, and recognition of this uncommon condition, when it occurs in an atypical location such as breast, is extremely difficult. The research aims to report a case of Rosai-Dorfman breast in a woman aged 37, with ill-defined mass in the left breast. Highlighting the immunohistochemical study of biopsy material, and it diffused expression for S-100 protein in histiocytic cells of the parenchyma.
\end{abstract}

Keywords: Rosai-Dorfman; Sinus histiocytosis; Breast

\section{Introduction}

The sinus histiocytosis with massive lymphadenopathy, described in 1969 by Rosai and Dorfman, [1] is a benign, self-limited disease characterized by diffuse lymph node involvement usually accompanied by fever, leukocytosis with neutrophilia and polyclonal hypergammaglobulinemia [2]. Lymphadenopathy usually is bilateral, painless and massive, and may also affect other lymphatic chains. It is observed in any age, but there is a predilection for young adult males

\footnotetext{
Manuscript accepted for publication June 6, 2013

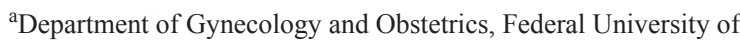
Amazon, UFAM, Manaus, Amazon, Brazil

${ }^{\mathrm{b}}$ Department of Mastology, Gynecology and Obstetrics, Federal University of Amazon, UFAM, Manaus, Amazon, Brazil

${ }^{\mathrm{c} C}$ Corresponding author: Thais Helena de Souza Silotti, Avenue Efigenio Sales n.200, Manaus, Amazon, Brazil. Email: thais_hrs@hotmail.com

doi: http://dx.doi.org/10.4021/jmc1351w
}

(1.4:1) and commonly, the disease occurs in whites and blacks, and rarely in Asians [3].

The extranodal involvement is observed in rare cases, and it is observed as typical sites of involvement, the skin and subcutaneous tissue, the upper airway, salivary glands, the eyeball, bones, central nervous system and breast, the latter being an uncommon site of presentation [4]. In most patients, the lesions regress spontaneously, however, these patients may develop recurrent, persistent disease, however stable or progressive disease leading to death in a few cases [5]. Systemic changes and involvement of multiple organs apparently indicate a poor prognosis.

The differential diagnosis of lesions of Rosai-Dorfman disease of the breast is very complex and important in view that one should rule out a variety of malignant lesions. According to the literature, the radiologically Rosai-Dorfman disease manifests itself as lesions poorly defined borders without calcifications, difficult to distinguish from a carcinoma, [6] which is actually a challenge since the doctor suspected until the therapeutic decision.

The authors of this study present a case of Rosai-Dorfman breast with a literature review on the topic, addressing different aspects of diagnosis and treatment.

\section{Case Report}

Woman, mulatto, 37 years old, married, domestic Brazilian sought medical treatment with a history of nodulation in the left breast, slightly painful and itchy, no redness, no report of preview drainage and with the same skin coloring. She was detected 2 years ago during self-breast examination, leading her to seek medical attention.

On physical examination, patients with good general condition, absence of peripheral lymphadenopathy. There was a palpable nodule in the left breast, in the union of the lower quadrants, measuring about $3 \mathrm{~cm}$ in greatest diameter, hardened, alteration without skin color, not adhered to the deep plane and painless. There were exams pulmonary, cardiac, abdominal and gynecological pathology, without semiological signs. Laboratory tests such as complete blood count and electrolytes without changes. 


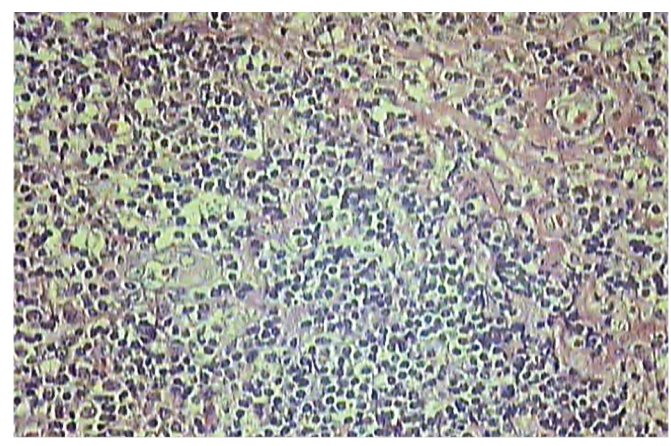

Figure 1. Photo microscopy of breast lesion, showing intense chronic inflammatory infiltrate composed of plasma cells, histiocytes and lymphocytes (Hematoxylin-eosin staining, with $100 \times)$.

Mammography was performed, which revealed no satisfactoriness for evaluation (BI-RADS 0). The research was complemented with breast ultrasound, which showed solid nodule and heterogeneous, with images circumscribed and irregular, complete with halo and thick $3.5 \times 1.5 \times 2.0 \mathrm{~cm}$ (BI-RADS/US IVc).

Core biopsy performed with a probable diagnosis of chronic mastitis abscess, and then a biopsy. The result of pathologic analysis of the lesion showed no growth with neoplastic chronic inflammatory process and outline of noncaseating granuloma, and complementary diagnosis by immunohistochemical assessment of the same specimen showed intense chronic inflammatory infiltrate composed of plasma cells, histiocytes and lymphocytes (Fig. 1), observing further proliferation of histiocytic cells with characteristics of eosinophilic cytoplasm and large vesicular nuclei with nucleoli sometimes evident, the presence of inflammatory cells pre-

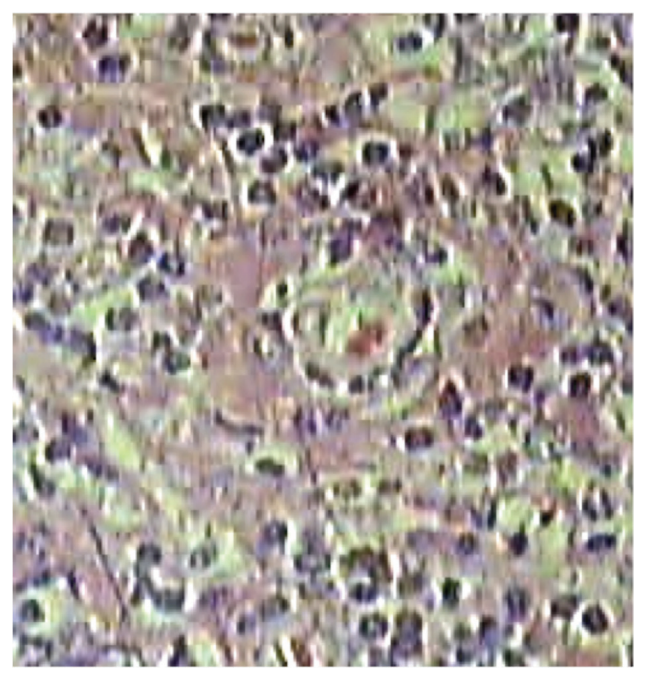

Figure 2. It is observed intact lymphocytes within the histiocyte, undigested, a phenomenon called Emperipolesis (Hematoxylin-eosin staining, with $400 \times$ ).

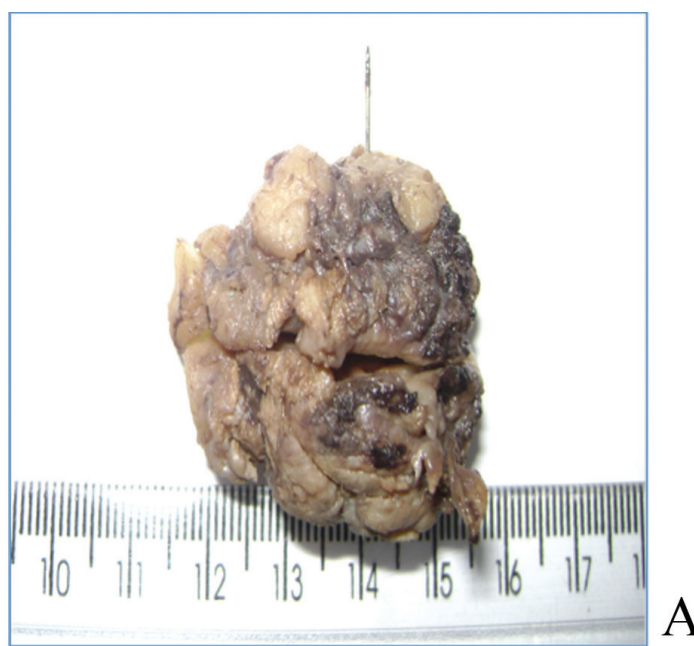

A

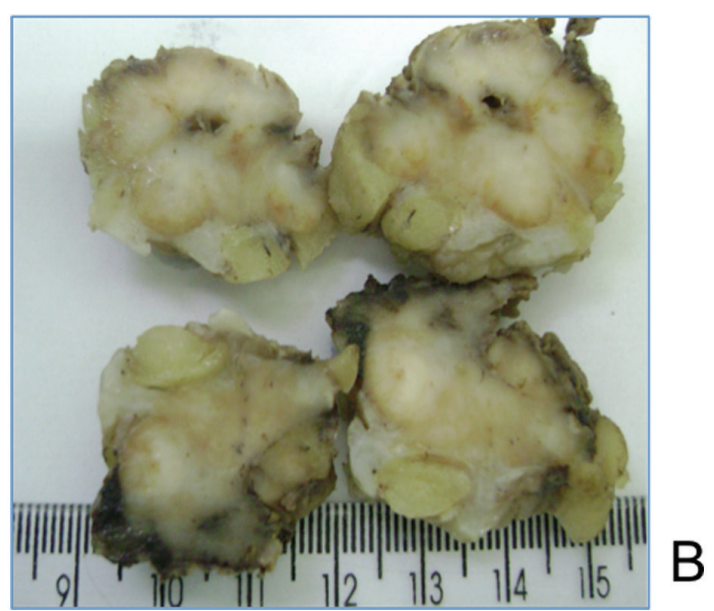

Figure 3. (a, b). Macroscopic surgical specimen; whitishyellowish, with brownish areas in between, jagged edges and asymmetrical weighing $15.58 \mathrm{~g}$.

served inside the cytoplasm of these histiocytic cells, with clear halo around them, a phenomenon emperipolesis named (Fig. 2). The immunohistochemical study revealed diffuse expression for protein S-100 in histiocytic cells, and CD20 ,$+ \mathrm{CD} 3+, \mathrm{CD} 163+, \mathrm{CD} 138+$ and negative for CD1a, which are morphological findings consistent with Rosai-Dorfman breast (extranodal).

Despite being a self-limiting disease, discuss with the patient the procedure to be performed, and because of the discomfort and anxiety, the patient opted for surgical excision of the lesion.

There was performed with wide resection specimen measuring $4.5 \times 4.0 \times 3.0 \mathrm{~cm}$, compact, showing whitishyellowish, with brownish areas in between, jagged edges and asymmetrical weighing 15.58 g (Fig. 3). Microscopically, there was diffuse inflammatory infiltration and histiocytic lympho-plasmacytic arranged around the mammary ducts 
Table 1. Reported Cases of Rosai-Dorfman Disease in the Breast

\begin{tabular}{ll}
\hline Author/year & Number of cases \\
\hline Perez/1993 & 2 cases \\
Hammond/1996 & 1 case \\
Soares/1999 & 1 case \\
Govender $/ 1997$ & 1 case \\
Green/1997 & 7 cases \\
Hummel/1999 & 1 case \\
Pham/2005 & 1 case \\
Ng SB/2000 & 1 case \\
Perera/2007 & 2 cases \\
Kuzmiak/2003 & 1 case \\
Picon/2010 & 1 case \\
Silotti/2012 & 1 case \\
\hline
\end{tabular}

and acini. The histiocytes showed ample clear cytoplasm sometimes with leukocytes, lymphocytes phagocytosed by setting emperipolesis.

Postoperatively the patient remained without complications and was discharged with 48 hours of hospitalization, designating themselves conservative management. The patient recovered without developing disease in the last 1 year of outpatient treatment.

\section{Discussion}

The Rosai-Dorfman is a clinical and pathological entity infrequent, have unknown etiology and is characterized by reactive changes of the immune system [4]. Its classical presentation is painless bilateral cervical lymphadenopathy in a young patient asymptomatic and usually evolves into a benign clinical course with low risk of systemic involvement.

In 1969, several years after its original description, it was felt that this was a disease process limited to lymph nodes. Currently has documented numerous cases in extranodal sites, which most often affect individuals with an age range a little higher, above 40 years. In another publication, we described seven cases with involvement of the mammary gland, showing the ages between 15 and 84 years, with an average of 42 years [7]. During the last 16 years, in the literature, there have been 19 cases of Rosai-Dorfman disease related to mammary gland, and of these, 18 women with a median age of 45 years and 1 case in a man of 23 years old, with palpable mass in the right breast [8] (Table 1).

Patients with involvement of the breast are rare and usually present with a palpable mass and ill-defined sensitivity exacerbated or an abnormal mammogram, typically of malignancy. The differential diagnosis based on sonographic and mammographic findings includes both benign and malignant solid masses. According to literature, ultrasound usually shows a hypoechoic mass vascularized, and usually mammography shows a mass with ill-defined borders, without calcifications [6].

The differential diagnosis based on histopathologic features include invasive breast carcinoma, Malakoplakia, diabetic mastopathy, fat necrosis, granulomatous mastitis, infectious granulomas, Erdheim-Chester, fibrous histiocytoma, malignant melanoma and Langerhans cell histiocytosis.

Definitive diagnosis is known thru the pathology, which can be established through fine needle aspiration (FNA), the core biopsy or surgical excision, histopathological characteristics that demonstrate similar regardless of the place of occurrence. The brand Rosai-Dorfman histiocytosis is a large, polygonal with round nuclei, vesicular chromatin, nuclear membrane thin, one to several eosinophilic nucleoli and abundant pale cytoplasm. Many of the histiocytes show emperipolesis, which signals useful feature, but not diseasespecific and can be found in other pathologies. However, emperipolesis is often more difficult to be viewed in that extra nodal lesions in the lymph nodes.

Lymph nodes show capsular fibrosis and dilated nodal sinuses filled with foamy histiocytes, hence the disease is also 
known as sinus histiocytosis with massive lymphadenopathy [9]. The immunohistochemical profile and is characterized by classic ultramicroscopic S-100+, CD1a, without Birbeck granules. This type of spectrum immunohistochemical suggests that sinus histiocytosis with massive lymphadenopathy is a proliferation of interdigitating dendritic cells in lymph nodes of antigen processing [3].

\section{Conclusion}

Currently there is a worldwide consensus of when to initiate treatment, or if conservative treatment is indicated, expectant or surgical. However, we know that Rosai-Dorfman disease usually evolves with spontaneous resolution in most cases. However, if you have documented, which may be persistent and aggressive evidence multisystemic, which can even be deadly in rare cases [2]. What can be observed in the literature, is that patients with bilateral breast show an indolent clinical course similar to that seen in other extranodal site [1]. Some unfavorable prognostic factors are: the extensive lymph node spread, extranodal involvement, immune disorders such as autoimmune hemolytic anemia, the presence of rheumatoid factor or antinuclear antibodies and the association of anemia with neutrophilia and lymphocytopenia [2].

There are no standardized guidelines on follow-up of patients with disease confined to the breast, however, must be graded individually in order to delete the systematization of disease or local recurrence [3]. However, we can conclude, as proposed by Landim et al [10], that tumors located exclusively in single anatomical site skin with surgical resection feasible, surgery should be considered: 1) tumors that persist or recur; 2) when the histopathologic findings are inconclusive; 3 ) the presence of symptoms; 4) if is the patient's wish. Complete resection with clear margins should be the goal of surgery is a great option for regional control.

\section{Conflict of Interest}

Authors declare no conflict of interest.

\section{Author's Contributions}

Thais Helena de Souza Silotti, substantial contributions to conception and design, acquisition of data, analysis and interpretation of data, drafting the article and critical revision of the article, final approval of the version to be published.

Maria Riselda Vinhote da Silva, substantial contributions to acquisition of data, analysis and interpretation of data, drafting the article and critical revision of the article, final approval of the version to be published.
Nilton Cesar O. Bessa, substantial contributions to acquisition of data, analysis and interpretation of data, drafting the article, final approval of the version to be published.

Ione Rodrigues Brun, substantial contributions to analysis and interpretation of data, critical revision of the article, final approval of the version to be published.

Livia Moura, substantial contributions to acquisition of data, analysis and interpretation of data, drafting the article and critical revision of the article, final approval of the version to be published.

\section{References}

1. Rosai J, Dorfman RF. Sinus histiocytosis with massive lymphadenopathy. A newly recognized benign clinicopathological entity. Arch Pathol. 1969;87(1):63-70.

2. Ng SB, Tan LH, Tan PH. Rosai-Dorfman disease of the breast: a mimic of breast malignancy. Pathology. 2000;32(1):10-15.

3. Brenn T, Calonje E, Granter SR, Leonard N, Grayson $\mathrm{W}$, Fletcher CD, McKee PH. Cutaneous rosai-dorfman disease is a distinct clinical entity. Am J Dermatopathol. 2002;24(5):385-391.

4. Foucar E, Rosai J, Dorfman R. Sinus histiocytosis with massive lynfadenopathy (Rosai-Dorfman disease): review of the entity. Semim Diagn Pathol 1990;7:19-73.

5. Warnke RA, Weiss LM, Chan JKC, et al. Tumors of the lymph nodes and spleen. In: Armed Forces Institute of Pathology, Atlas of tumor pathology, 3rd ed., fasc. 14. Washington, DC: AFIP,1995 : 349.

6. Ferrando J, Marti-Laborda RM. Histiocytosis in skin manifestations of systemic diseases in pediatrics. En: Fonseca-Jarpyo E, editor. Diagnostic and therapeutic protocols in pediatric dermatology (in Spanish). Madrid: Classroom Medical Group;1993.pp.159-165.

7. Paire V, Bossard C, Vabres B, Weber M, Pechereau A. [Orbital locations in Rosai-Dorfman disease: a series of three consecutive cases]. J Fr Ophtalmol. 2008;31(7):673-682.

8. Picon-Coronel G, Palmerin-Bucio ME, Mendez-Perez V, Alvarado-Cabrero I. [Mammary gland Rosai Dorfman disease. A case report and literature review]. Gac Med Mex. 2010;146(3):212-215.

9. Lu CI, Kuo TT, Wong WR, Hong HS. Clinical and histopathologic spectrum of cutaneous Rosai-Dorfman disease in Taiwan. J Am Acad Dermatol. 2004;51(6):931939.

10. Landim FM, Rios HO, Costa CO, Feitosa RGF, Rocha Filho FD, Costa AAA. Rosai-Dorfman Cutaneous disease: a case report and review of literature (in Portuguese). An Bras Dermatol. vol.84 no.3 Rio de Janeiro 2009. pp.2-4. 